\title{
Prática de corrida de rua melhora estados de humor e auto avaliação do sono
}

\author{
Running practice improves mood state and sleep self-assessment \\ La práctica de correr en la calle mejora el estado de ánimo y la autoevaluación del sueño
}

Recebido: 16/10/2021 | Revisado: 24/10/2021 | Aceito: 30/10/2021 | Publicado: 01/11/2021

\author{
Bianca Caroline Dezordi \\ ORCID: https://orcid.org/0000-0002-2874-1703 \\ Universidade Estadual de Maringá, Brasil \\ E-mail: bianca.dezordi@ hotmail.com \\ Karina Alves da Silva \\ ORCID: https://orcid.org/0000-0003-2206-8543 \\ Universidade Estadual do Oeste do Paraná, Brasil \\ E-mail: kaalvessilvaedfisica@gmail.com \\ Lilian Keila Barazetti \\ ORCID: https://orcid.org/0000-0002-5694-9835 \\ Universidade Estadual do Oeste do Paraná, Brasil \\ E-mail: lilianbarazetti@gmail.com \\ Ricardo Brandt \\ ORCID: https://orcid.org/0000-0001-6018-0347 \\ Universidade Estadual do Oeste do Paraná, Brasil \\ E-mail: ricabrandt@gmail.com
}

\begin{abstract}
Resumo
As variáveis sono e os estados de humor são aspectos psicológicos influenciados pela prática de exercício físico, dessa forma, o objetivo da pesquisa foi investigar o efeito de 16 semanas de prática de corrida em relação à auto avaliação do sono e às mudanças nos estados de humor. Participaram da pesquisa 18 corredores recreacionais (13 Mulheres; 5 Homens). A Escala de Humor de Brunel (BRUMS) avaliou os Estados de Humor, sendo aplicado antes e depois do treino, já a questão autorreferida sobre o sono foi feita apenas antes do treino. Aplicou-se estatística descritiva e inferencial para avaliar as relações entre sono, humor e corrida, as diferenças foram consideradas estatisticamente significativa quando $\mathrm{p}<0.05$. Os resultados apresentaram 5 das variáveis do humor raiva, tensão, depressão, confusão e fadiga apresentaram mudanças significativas pré e pós intervenção, já o vigor não se alterou. $\mathrm{O}$ tamanho do efeito mostrou uma grande magnitude na redução da raiva e depressão, moderada redução da tensão e confusão, insignificante redução do vigor e um aumento pequeno na magnitude da fadiga. Com relação a auto avaliação do sono, não se verificou diferença significativa pré e pós intervenção. Pode-se concluir que a prática de corrida de rua em intensidade moderada pode melhorar a qualidade do sono, e a manutenção dos níveis adequados de humor.
\end{abstract}

Palavras-chave: Exercício; Corrida; Humor; Sono.

\begin{abstract}
Sleep and mood states are psychological aspects influenced by the practice of physical exercise, thus, the objective of the research was to investigate the effect of 16 weeks of running in relation to the sleep self-assessment and changes in the states of humor. Eighteen recreational runners (13 Women; $5 \mathrm{Men}$ ) participated in the research. The Brunel Mood Scale (BRUMS) assessed Mood States, being transported before and after training, while the self-reported question about sleep was done just before training. Descriptive and inferential statistics were applied to assess the relationships between sleep, mood and running, differences were considered statistically significantly when $\mathrm{p}<0.05$. The results showed 5 dissipate from the mood variables anger, tension, distortion, confusion and fatigue, dissipate changes before and after the intervention, while vigor did not change. The effect size showed a large magnitude in reduction of anger and depression, moderate reduction in tension and confusion, negligible reduction in vigor, and a small increase in magnitude of fatigue. Regarding sleep self-assessment, there was no difference before and after the intervention. It can be observed that a moderate intensity street running practice can improve sleep quality and maintain adequate levels of mood.
\end{abstract}

Keywords: Exercise; Race; Humor; Sleep.

\section{Resumen}

Las variables sueño y estados de ánimo son aspectos psicológicos influenciados por la práctica de ejercicio físico, por lo que el objetivo de la investigación fue investigar el efecto de 16 semanas de carrera en relación a la autoevaluación del sueño y cambios en los estados de humor. Dieciocho corredores recreativos (13 mujeres; 5 hombres) participaron en la investigación. La Brunel Mood Scale (BRUMS) evaluó los estados de ánimo, siendo transportados antes y después del entrenamiento, mientras que la pregunta autoinformada sobre el sueño se realizó justo antes del 
entrenamiento. Se aplicaron estadísticas descriptivas e inferenciales para evaluar las relaciones entre el sueño, el estado de ánimo y la carrera, las diferencias se consideraron estadísticamente significativas cuando $\mathrm{p}<0.05$. Los resultados 5 se disipan de las variables del estado de ánimo ira, tensión, distorsión, confusión y fatiga, disipan los cambios antes y después de la intervención, mientras que el vigor no cambió. El tamaño del efecto mostró una gran magnitud en la reducción de la ira y la depresión, una reducción moderada de la tensión y la confusión, una reducción insignificante del vigor y un pequeño aumento en la magnitud de la fatiga. En cuanto a la autoevaluación del sueño, no hubo diferencia antes y después de la intervención. Se puede observar que una práctica de carrera callejera de intensidad moderada puede mejorar la calidad del sueño y mantener niveles adecuados de humor.

Palabras clave: Ejercicio; Raza; Humor; Dormir.

\section{Introdução}

O exercício físico influencia diversos fatores do corpo humano, fisiológicos e psicológicos (Ali, Aseem \& Hussain 2019), entre eles o sono e os estados de humor (Brandt et al., 2016; Brandt, Bevilacqua \& Andrade 2017). O sono é um importante processo de recuperação no esporte de resistência, que é caracterizado por demandas físicas prolongadas e extenuantes que podem influenciar aspectos fisiológicos e mecânicos, além do comportamento emocional e psicossocial dos atletas (Andrade et al., 2018). Nesse contexto, a qualidade do sono dos atletas está associada, entre outros aspectos, ao tempo gasto na cama, ao tempo para adormecer, ao acordar não mais de uma vez por noite e à obtenção de sono restaurador adequado (Andrade et al., 2018; Ohavon et al., 2017).

Desta forma, o sono é importante no processo de recuperação, sendo que os distúrbios associados a ele são comumente evidenciados após treinamento intenso ou em competição, podendo afetar o processo de recuperação e as funções cognitivas (Nédélec et al., 2013). Mesmo sendo constantemente enfatizada a sua importância, muitos atletas têm uma quantidade e qualidade insuficiente de sono habitual (Gupta, Morgan \& Gilchrist 2017). O sono apresenta dois estados básicos, baseados em parâmetros fisiológicos: sem movimentos oculares rápidos (NREM) e o sono com movimentos oculares rápidos (REM), funcionando como um continuum de totalmente acordado a sono profundo (Doherty et al., 2019).

Uma qualidade ruim do sono, também tem sido associada a distúrbios de humor (Dinges et al., 1997), sendo que diversas pesquisas têm encontrado relação significativa entre o sono e os estados de humor (Brandt, Bevilacqua \& Andrade 2017; Lastella, Lovell \&Sargent 2014), ou seja, o sono torna-se um fator importante na recuperação emocional de atletas (Kölling et al., 2015).

Estudos empíricos e de revisão de literatura demonstram a atenção dada aos estados de humor em atletas de vários esportes e níveis competitivos (Brandt et al., 2017; Harris et al., 2015; Killer et al., 2015). O humor é frequentemente temporário e pode variar em função da intensidade e duração do exercício (Lane \& Terry 2000). Os pesquisadores determinaram que o humor compreende alto vigor e níveis mais baixos de tensão, depressão, raiva, fadiga e confusão está associado ao desempenho ideal. Pesquisas recentes (Han, Parsons-Smith \& Terry 2020; Quartoli et al., 2018) têm demonstrado que podem existir outros modelos de humor, com características distintas, do tradicional "perfil de iceberg" proposto por Morgan (1980), denominados iceberg, inverse Everest, inverse iceberg, shark fin, submerged, e surface profiles.

Os estados de humor têm colaborado com o monitoramento das adaptações dos treinamentos, além de avaliar o risco de burnout de atletas e praticantes de esportes, assim como monitorar a recuperação e o excesso de treinamento (Parsons-Smith et al., 2017).

Embora, compreensivelmente, sono e humor estejam inter-relacionados, muitas perguntas sobre os efeitos de direção e causalidade entre sono e humor permaneceram sem resposta (Hamilton et al., 2007). Portanto a presente pesquisa tem como objetivo apresentar resultados em referentes à prática de corrida de rua como um exercício ótimo nos estados de humor e na auto avaliação do sono de quem pratica, assim, ao identificar essa relação o estudo pode auxiliar treinadores a compreender a influência da corrida de rua nos estados de humor e sono. 


\section{Metodologia}

\section{Tipo de pesquisa}

Trata-se de uma pesquisa longitudinal com intervenção em indivíduos praticantes de corrida de rua, do tipo quantitativa e descritiva.

\section{População e amostra}

Participaram da pesquisa 18 corredores recreacionais (média de idade de 40 anos, DP=10; entre 23 e 58 anos), dentre eles 5 homens (média de idade 38 anos, DP = 9.0) e 13 mulheres (média de idade 41 anos, DP=10.6). Todos os corredores compartilhavam do mesmo treinador que supervisionava cada sessão de treino.

\section{Critérios de inclusão e exclusão}

Os indivíduos praticavam o esporte há mais de 5 anos e participavam de competições regionais. Foram determinados como critérios de exclusão: (a) a presença de alguma doença cardiovascular, (b) ter faltado mais de 3 sessões consecutivas devido à alguma lesão ou doença, e (c) ter realizado menos de $90 \%$ das sessões de treinamento conforme especificado na proposta do programa.

\section{Instrumentos}

\section{Estados de Humor}

O questionário BRUMS, utilizado para coletar os Estados de Humor, foi traduzido para o Português e validados para a população brasileira por Rohlfs et al. (Brandt et al., 2016). O instrumento apresenta 24 itens avaliados em uma escala de 5 pontos, de 0 (nada) até 4 (extremamente), sendo o padrão de referência é de "como você está se sentindo agora?". O BRUMS também compreende 6 subescalas (tensão, depressão, raiva, vigor, fadiga e confusão), cada uma delas consiste em 4 descrições de humor, sendo assim apresentam uma pontuação de 0 à 16 . Os valores de consistência interna (alfa de Cronbach) para cada uma das 6 subescalas e toda a escala combinada foram todos maiores que 0,76 no estudo de Rohlfs et al. (2006). Em termos da natureza de cada estado de humor (Brandt et al., 2016).

Tensão - definido por sentir-se nervoso, ansioso, em pânico e preocupado.

Depressão - definido por sentir-se abatido, desanimado, infeliz e miserável é o humor deprimido, em vez de um estado clínico de depressão.

Raiva - definido por sentir-se irritado, zangado, mal-humorado e amargo.

Vigor - fator definido por sentir-se ativo, animado, enérgico e alerta.

Fadiga - definido por sentir-se sonolento, exausto, cansado e esgotado.

Confusão - definido pelo sentimento de confusão e incerteza.

Sono

A pergunta sobre a auto avaliação do sono foi "Como você avalia a qualidade do seu sono nos últimos dias?". Os participantes classificaram a qualidade do sono em uma escala do tipo Likert da seguinte forma: $1=$ muito ruim, $2=$ ruim, $3=$ normal, $4=$ bom e $5=$ excelente. Também registramos a idade dos participantes, modalidade esportiva (individual ou coletiva) e anos de prática em seus esportes. Nosso uso da qualidade do sono autorreferida foi baseado em estudos anteriores, que também investigaram a qualidade do sono dessa maneira (Brandt et al., 2017). 


\section{Procedimentos éticos}

O protocolo do estudo foi aprovado pelo Comitê de Ética em Pesquisa da Universidade Estadual do Oeste do Paraná (5231219.9.0000.0107). Os dados foram coletados durante os 10 minutos imediatamente anteriores ao primeiro treinamento $\mathrm{e}$ do último treinamento. Os atletas foram abordados pelos pesquisadores e encaminhados para um local específico e tranquilo dentro do Complexo Esportivo da Universidade Estadual Oeste do Paraná onde realizaram as intervenções de humor e sono sob a supervisão de dois pesquisadores. Toda a coleta de dados foi padronizada em termos de condições de privacidade e supervisão. Os participantes foram solicitados a serem os mais sinceros possível ao preencher os instrumentos de humor e sono, portanto, foram instruídos a responder em relação aos seus sentimentos atuais. Por fim, o tempo total de coleta de dados (tempo de deslocamento até o local de coleta e conclusão do instrumento) foi de aproximadamente dez minutos para cada participante.

\section{Análise Estatística}

As análises estatísticas foram realizadas a partir do software SPSS versão 20.0 (IBM Corporation, Armonk, NY). Os dados foram apresentados em valores de frequência, mediana e intervalo interquartis. A normalidade foi verificada a partir do teste Shapiro-wilk. Para comparar as mudanças no comportamento do sono e nos estados de humor pré e pós intervenção aplicou se o teste de Wilcoxon. O tamanho de efeito foi calculado de acordo com Cohen, para determinar o significado da diferença na comparação entre pares. A magnitude de $d$ foi classificada como pequena $(d=0,2)$, moderada $(d=0,5)$ e grande $(d=0,8)(13)$. Todas as diferenças são consideradas estatisticamente significativas quando $p<0,05$.

\section{Resultados}

Os sujeitos seguiram uma planilha de treinamento de corrida programada durante 16 semana, planejadas por um profissional experiente na área (Tabela 1). 
Tabela 1. Caracterização das sessões de treino durante as 16 semanas do estudo.

\begin{tabular}{|c|c|c|c|c|c|c|c|}
\hline $\begin{array}{r}\text { Micro } \\
\text { ciclo }\end{array}$ & $\begin{array}{c}\mathbf{N}^{\mathbf{0}} \\
\text { sessões }\end{array}$ & $\begin{array}{c}\text { Sessão } \\
\text { (1) }\end{array}$ & $\begin{array}{c}\text { Sessão } \\
\text { (2) }\end{array}$ & Intensidade & $\begin{array}{c}\text { Menor } \\
\text { volume } \\
\text { seminal } \\
(\mathbf{k m})\end{array}$ & $\begin{array}{c}\text { Maior } \\
\text { volume } \\
\text { semanal } \\
(\mathbf{k m})\end{array}$ & $\begin{array}{l}\text { Carga total de } \\
\text { treino }(\mathbf{k m})\end{array}$ \\
\hline & & Método & Método & & & & \\
\hline 1 & 2 & VA & VA & $\mathrm{BA} / \mathrm{MO}$ & 4 & 4 & 8 \\
\hline 2 & 2 & VA & VA & $\mathrm{BA} / \mathrm{MO}$ & 4.5 & 4.5 & 9 \\
\hline 3 & 2 & VA & VA & $\mathrm{BA} / \mathrm{MO}$ & 4.5 & 4.5 & 9 \\
\hline 4 & 2 & VA & VA & $\mathrm{BA} / \mathrm{MO}$ & 5 & 5 & 10 \\
\hline 5 & 2 & VA & VA & $\mathrm{BA} / \mathrm{MO}$ & 3.5 & 4 & 7.5 \\
\hline 6 & 2 & VA & VA & $\mathrm{BA} / \mathrm{MO}$ & 4.5 & 5 & 9.5 \\
\hline 7 & 2 & $\mathrm{EC}$ & $\mathrm{EC}$ & MO & 5 & 7 & 12 \\
\hline 8 & 2 & VA & VA & MO/AL & 4 & 4.5 & 8.5 \\
\hline 9 & 2 & VA & VA & MO/AL & 4 & 8 & 12 \\
\hline 10 & 2 & $\mathrm{EC}$ & $\mathrm{EC}$ & BA & 3 & 3.5 & 6.5 \\
\hline 11 & 2 & VA & VA & $\mathrm{MO} / \mathrm{AL}$ & 5 & 5 & 10 \\
\hline 12 & 2 & VA & VA & $\mathrm{MO} / \mathrm{AL}$ & 4.5 & 5 & 9.5 \\
\hline 13 & 2 & VA & VA & MO/AL & 5.5 & 5.5 & 11 \\
\hline 14 & 2 & VA & VA & MO/AL & 5 & 6 & 11 \\
\hline 15 & 2 & $\mathrm{EC}$ & $\mathrm{EC}$ & MO/AL & 5 & 5 & 12 \\
\hline 16 & 2 & $\mathrm{EC}$ & $\mathrm{EC}$ & BA & 3.5 & 4 & 7.5 \\
\hline
\end{tabular}

EC: extenso contínuo; VA: variacional; BA: baixo; MO: moderado; AL: alto.

Fonte: próprios autores (com base nos resultados da pesquisa).

Ao longo das 16 semanas de treinamento a maior parte dos indivíduos participantes, auto avaliou o sono com respostas entre regular e bom. Dessa forma, apesar de não haver diferenças estatísticas nos resultados da autoavaliação do sono, ainda assim percebe-se que no decorrer das semanas de intervenção os sujeitos respondem em direção a uma melhor qualidade do sono (Tabela 2). 
Tabela 2. Auto avaliação do sono em corredores de rua após 16 semanas de prática.

\begin{tabular}{|c|c|c|c|c|c|c|c|c|c|c|c|}
\hline \multirow{2}{*}{$\begin{array}{l}\text { Sono } \\
\text { Semana }\end{array}$} & \multicolumn{2}{|c|}{$\begin{array}{l}\text { Muito } \\
\text { Ruim }\end{array}$} & \multicolumn{2}{|c|}{ Ruim } & \multicolumn{2}{|r|}{ Regular } & \multicolumn{2}{|c|}{ Bom } & \multicolumn{2}{|c|}{ Excelente } & \multirow[t]{2}{*}{ Total } \\
\hline & $\mathrm{f}$ & $\%$ & f & $\%$ & $\mathrm{f}$ & $\%$ & $\mathrm{f}$ & $\%$ & $\mathrm{f}$ & $\%$ & \\
\hline Sem. 01 & 0 & $0 \%$ & 0 & $0 \%$ & 5 & $27,7 \%$ & 10 & $55,5 \%$ & 3 & $16,6 \%$ & 18 \\
\hline Sem. 02 & 1 & $5,5 \%$ & 1 & $5,5 \%$ & 4 & $22,2 \%$ & 10 & $55,5 \%$ & 2 & $11,1 \%$ & 18 \\
\hline Sem. 03 & 1 & $5,5 \%$ & 5 & $27,7 \%$ & & $950 \%$ & 3 & $16,6 \%$ & 3 & $16,6 \%$ & 18 \\
\hline Sem. 04 & 1 & $5,5 \%$ & 0 & $0 \%$ & & $3 \quad 16,6 \%$ & 11 & $61,1 \%$ & 3 & $16,6 \%$ & 18 \\
\hline Sem. 05 & 1 & $5,5 \%$ & 0 & $0 \%$ & & $5 \quad 27,7 \%$ & 9 & $50 \%$ & 3 & $16,6 \%$ & 18 \\
\hline Sem. 06 & 0 & $0 \%$ & 2 & $11,1 \%$ & & $6 \quad 33,3 \%$ & 8 & $44,4 \%$ & 2 & $11,1 \%$ & 18 \\
\hline Sem. 07 & 0 & $0 \%$ & 1 & $5,5 \%$ & & $5 \quad 27,7 \%$ & 10 & $55,5 \%$ & 2 & $11,1 \%$ & 18 \\
\hline Sem. 08 & 0 & $0 \%$ & 1 & $5,5 \%$ & & $\begin{array}{ll}3 & 16,6 \%\end{array}$ & 10 & $55,5 \%$ & 4 & $22,2 \%$ & 18 \\
\hline Sem. 09 & 0 & $0 \%$ & 0 & $0 \%$ & & $4 \quad 22,2 \%$ & 11 & $61,1 \%$ & 3 & $16,6 \%$ & 18 \\
\hline Sem. 10 & 1 & $5,5 \%$ & 0 & $0 \%$ & & $5 \quad 27,7 \%$ & 9 & $50 \%$ & 3 & $16,6 \%$ & 18 \\
\hline Sem. 11 & 0 & $0 \%$ & 1 & $5,5 \%$ & & $4 \quad 22,2 \%$ & 9 & $50 \%$ & 4 & $22,2 \%$ & 18 \\
\hline Sem. 12 & 0 & $0 \%$ & 0 & $0 \%$ & & $4 \quad 22,2 \%$ & 10 & $55,5 \%$ & 4 & $22,2 \%$ & 18 \\
\hline Sem. 13 & 0 & $0 \%$ & 1 & $5,5 \%$ & & $5 \quad 27,7 \%$ & 8 & $44,4 \%$ & 3 & $16,6 \%$ & 18 \\
\hline Sem. 14 & 1 & $5,5 \%$ & 0 & $0 \%$ & & $5 \quad 27,7 \%$ & 9 & $50 \%$ & 3 & $16,6 \%$ & 18 \\
\hline Sem. 15 & 0 & $0 \%$ & 0 & $0 \%$ & & $6 \quad 33,3 \%$ & 9 & $50 \%$ & 3 & $16,6 \%$ & 18 \\
\hline Sem. 16 & 0 & $0 \%$ & 0 & $0 \%$ & & $4 \quad 22,2 \%$ & 11 & $61,1 \%$ & 3 & $16,6 \%$ & 18 \\
\hline
\end{tabular}

Nota: $*$ diferença

Fonte: próprios autores (com base nos resultados da pesquisa).

As variáveis relacionadas aos Estados de Humor foram avaliadas nos sujeito, pré e pós intervenção das 16 semanas de treinamento de corrida. Dessa forma, foram verificados resultados com valores apresentando diferenças estatisticamente significativas, principalmente com a redução nos níveis dos componentes: raiva ( $\mathrm{p}=0.000)$, tensão ( $\mathrm{p}=0.003)$, depressão ( $\mathrm{p}=0.000)$ e confusão ( $\mathrm{p}=0.004)$. Já em relação à fadiga, houve um aumento significativo que foi observado: fadiga ( $\mathrm{p}=0.039$ ). Apesar de diferenças observadas nos níveis de vigor, o resultado não obteve diferença estatisticamente significativa para ( $>0.05$ ) entre os momentos avaliados (Tabela 3). 
Tabela 3. Valores da mediana e quartis dos estados de humor dos corredores antes e após 16 semanas de treinamento.

\begin{tabular}{lcccc}
\hline Variáveis & M & Q1 & Q3 & $p$ \\
\hline Raiva pré & 0.68 & 0.21 & 1.10 & \\
Raiva pós & 0.06 & 0.00 & 1.14 & $0.000^{*}$ \\
Tensão pré & 1.43 & 0.79 & 2.70 & \\
Tensão pós & 0.71 & 0.17 & 1.40 & $0.003^{*}$ \\
Depressão pré & 0.90 & 0.42 & 0.90 & \\
Depressão pós & 0.18 & 0.04 & 0.18 & $0.000^{*}$ \\
Vigor pré & 8.84 & 7.37 & 9.26 & \\
Vigor pós & 8.40 & 7.12 & 9.56 & 0.602 \\
Fadiga pré & 2.25 & 1.54 & 3.65 & \\
Fadiga pós & 3.50 & 2.01 & 5.87 & $0.039^{*}$ \\
Confusão pré & 0.46 & 0.18 & 1.25 & $0.004^{*}$ \\
Confusão pós & 0.12 & 0.04 & 0.37 & \\
\hline
\end{tabular}

Fonte: próprios autores (com base nos resultados da pesquisa).

O tamanho de efeito mostrou uma grande magnitude na redução da raiva (ES=0.87) e depressão (ES=0.84), moderada na redução da tensão $(E S=0.69)$ e confusão $(E S=0.68)$, uma magnitude insignificante na redução do vigor $(E S=0.12)$ e um aumento pequeno na magnitude da fadiga $(\mathrm{ES}=0.48)$. Com relação ao sono nõ houve mudança significativa pré e pós intervenção (ES=0.07) (Figura 1).

Figura 1. Tamanho do efeito nas variáveis dos estados de humor e sono entre os corredores pós treino.

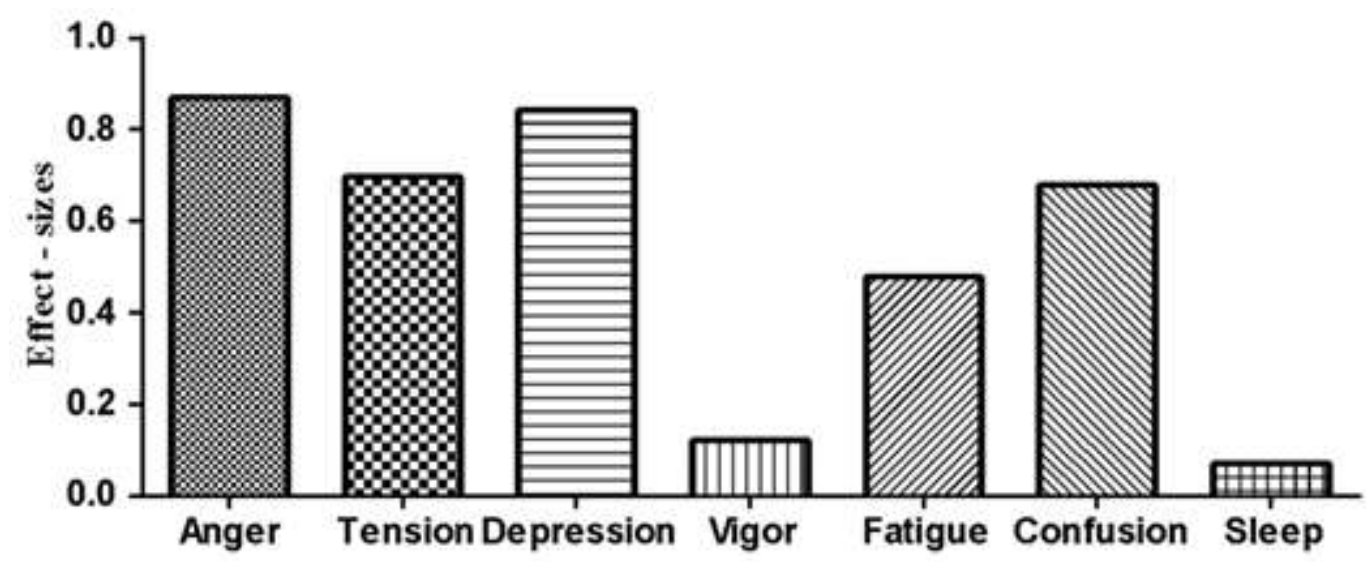

Fonte: próprios autores (com base nos resultados da pesquisa). 


\section{Discussões}

O objetivo deste estudo foi investigar o efeito de 16 semanas de treinamento de corrida de rua em relação a sono e humor de corredores recreacionais. Até onde sabemos, este é o primeiro estudo a investigar o comportamento do humor e sono ao longo de um programa de um treinamento sistematizado de corrida. Os achados demonstram que as sessões promoveram alterações significativas nos estados de humor, além de uma discreta melhora gradual na percepção da qualidade do sono.

Com o aumento e a popularidade da corrida de rua, há uma crescente necessidade em se investigar os efeitos da prática sobre o sono e humor, sendo que eles vem sendo apontando como importantes elementos relacionados a performance e recuperação (Andrade et al., 2018; Brandt, Bevilacqua \& Andrade 2017; Quartiroli et al., 2018). Devido às limitadas informações em praticantes de exercícios aeróbios, nossas descobertas podem fornecer uma valiosa contribuição do gerenciamento desses parâmetros em corredores recreacionais.

Os resultados dos estados de humor pré e pós treinamento demonstram que houve reduções significativas nos componentes raiva, tensão, depressão e confusão. Por outro lado, a fadiga apresentou aumento significativo. Tais achados estão de acordo com demais investigações nas quais observou-se mudanças semelhantes nas dimensões do humor a partir do exercício aeróbio agudo (Brellenthin et al.,2017; Lastella, Lovell \& Sargent 2014; McDoewll, Campbell \& Herring 2016). Boa saúde mental está relacionada aos modelos iceberg, surface ou shark fin (modelos de curvas) que apresentam escores reduzidos para os componentes tensão, depressão, raiva, fadiga e confusão e alto escore do componente vigor (Ohavon et al., 2017; Parsons-Smith, Terry, Machin 2017; Han, Parsons-Smith \&Terry 2020).

A grande magnitude dos tamanhos de efeito verificada nas dimensões da raiva e depressão e moderada na tensão e confusão se assemelham aos achados anteriores (McDowell, Campbell \& Herring 2016; Brandt et al., 2016) demonstrando uma melhora dos aspectos emocionais. Por outro lado, o pequeno tamanho de efeito da fadiga pode representar para a população estudada queda nos níveis de energia e esgotamento, podendo indicar leve sobrecarga do treinamento (Rodrigues et al., 2017). Esse resultado já era esperado em virtude do estresse metabólico gerado pelo aumento progressivo da intensidade das cargas ao longo das 16 semanas, corroborando achados anteriores conduzidos a partir de cargas moderadas e de alta intensidade (Rohlfs 2006; Evangelista et al., 2017).

Vários fatores podem influenciar os efeitos do exercício físico no humor, como as características dos participantes, o estímulo ao exercício, além de fatores neurobiológicos e psicológicos (Lastella, Lovell \& Sargent 2014). Nesse sentido, ressaltamos que o aumento progressivo da intensidade de esforço ao longo das sessões de treino pode auxiliar a explicar os resultados da melhora dos estados de humor, uma vez que efeitos positivos têm sido relacionados a intensidades auto selecionadas, na qual a intensidade de esforços causa maior fadiga central e periférica, afetando consequentemente o humor (Dishman et al., 2006).

Embora os exercícios sejam capazes de influenciar os aspectos emocionais, podem contribuir também na qualidade do sono, que por sua vez, auxilia na melhor recuperação física e emocional dos atletas (Killer et al., 2017). Apesar de não ser observada alterações significativas na percepção da qualidade do sono após as 16 semanas de treinamento, a discreta melhora deste demonstra estar relacionada à intensidade de esforço das sessões. No geral, as sessões compostas por cargas de moderada a alta intensidade promoveram uma melhor percepção da qualidade do sono em comparação às de baixa intensidade, demonstrando que o exercício agudo mais intenso pode ser um fator determinante (Teixeira et al., 2016). Ademais, é importante acrescentar que a leve inclinação à melhora do comportamento do sono pode ter contribuído, ainda que pouco, na manutenção dos níveis adequados nos estados de humor. 


\section{Conclusão}

A corrida de rua é uma modalidade que vem ganhando adeptos e com isso surge a necessidade de monitorar os impactos positivos e deletérios na dos praticantes. Essa pesquisa evidenciou a mudanças de humor antes e depois da prática de corrida de rua, assim como efeitos na auto avaliação do sono. Praticar corrida de rua em intensidade moderada e um fator que pode contribuir na manutenção dos níveis adequados de humor, sendo positivo para a saúde física e mental.

\section{Referências}

Ali, K., Aseem, A., \& Hussain M. (2019). Anaerobic training and its effects on sleep quality, state, and trait anxiety in collegiate athletes. Sport Sciences for Health 15: 453-461. https://doi.org/10.1007/s11332-019-005531

Andrade, A., Bevilacqua, G. G., Casagrande, P., Brandt, R., \& Coibra, D. (2018). Sleep quality associated with mood in elite athletes. The Physician an Sportsmedicine; 47:312-317. https://doi.org/10.1080/00913847.2018.1553467

Andrade, A., Bevilacqua, G. G., Coimbra, D. R., Pereira, F. S., \& Brandt, R. (2016). Sleep Quality, Mood and Performance: A Study of Elite Brazilian Volleyball Athletes. Journal of Sport Science and Medicine; 15: 601-605.

Brandt, R., Bevilacqua, G. G., \& Andrade, A. (2017). Perceived Sleep Quality, Mood States, and Their Relationship with Performance Among Brazilian Elite Athletes During a Competitive Period. Journal of Strength and Conditional Research; 31(4):1033-1039. https://doi.org/10.1519/JSC.0000000000001551

Brandt, R., Herrero, D., Massetti, Crocetta, T. B., Guarnieri, R., Monteiro, C. B. M., Viana, M. S., Bevilacqua, G. G., Abreu, L. C., \& Andrade, A. (2016). The Brunel Mood Scale Rating in mental health for physically active and apparently healthy populations. Health; 8: 125-132. https://doi.org/ 10.4236/health.2016.82015

Brandt R., Liz, C. M., Crocetta, T. B., Arab, C., Bevilaqua, G. G., Dominski, F. H., Vilarino, G. T., \& Andrade, A. (2014). Mental health and associated factors in athletes during the open games of Santa Catarina. Revista Brasileira de Medicina do Esporte; 29: 276-280. https://doi.org/10.1590/151786922014200401607 .

Brellenthin, A. G., Crombie, K. M., Hillard, C. J., \& Koltyn, K. F. (2017). Endocannabinoid and Mood Responses to Exercise in Adults with Varying Activity Levels. Medicine and Science in Sports and Exercise; 2: 138-145. https://doi.org/10.1249/MSS.0000000000001276

Dinges, D. F., Pack, F., Williams, K., Gillen, K. A., Powell, J. W., Ott, G. E., Aptowicz, C., \& Pack, A. I. (1997). Cumulative sleepiness, mood disturbance, and psychomotor vigilance performance decrements during a week of sleep restricted to 4-5 hours per night. Sleep; 20 : $267-277$.

Dishman, R. K., Berthoud, H. R., Booth, F. W., Cotman, C. W., Edgerton, V. R., Fleshner, M. R., Gandevia, S. C., Gomez-Pinilla, F., Greenwood, B. N., Hillman, C. H., Kramer, A. F., Levin, B. E., Moran, T. H., Russo-Neustadt, A. A., Salamone, J. D., Hoomissen, J. D. V., Wade, C. E., York, D. A., \& Zigmond, M. J. (2006). Neurobiology of exercise. Obesity; 14, 345-356. https://doi.org/10.1038/oby.2006.46

Doherty, R., Madigan, S., Warrington, G., \& Ellis, J. (2019). Sleep and Nutritions Interactions: Implications for Athletes. Nutrients; 11, 822. https://doi.org/10.3390/nu11040822

Evangelista, A. L., Evangelista, R. A. G. T., Rica, R. L., Machado, A. F., Miranda, J. M. Q., Teixeira, C. V. L. S., Lopes, C. R., \& Bocalini, D. S. (2017). Effects of High-Intensity Calisthenic Training on Mood and Affective Responses. Journal of Exercise Physiology online; 20: 15-23.

Gupta, L., Morgan, K., \& Gilchrist, S. (2017). Does elite sport degrade sleep quality? A systematic review. Sports Medicine; 47:1317-1333. https://doi.org/10.1007/s40279-016-0650-6

Hamilton, N. A., Nelson, C. A., Stevens, N., \& Kitzman, H. (2007). Sleep and psychological well-being. Social Indicators Research; 82: 147-163. https://doi.org/10.1007/s11205-006-9030-1

Han, C. S. Y., Parsons-Smith, R. L., \& Terry, P. (2020). Mood profiling in Singapore: cross-cultural validation and potential applications of mood profile clusters. Frontiers in Psychology; 11:665. https://doi.org/10.3389/fpsyg.2020.00665

Harris, A., Gundersen, H., Mork-Andreassen, P., Thun, E., Bjorvatan, B., \& Pallesen, S. (2015). Restricted use of electronic media, sleep, performance, and mood in high school athletes-a randomized trial. Sleep Health; 4: 314:321. https://doi.org/10.1016/j.sleh.2015.09.01

Hausswirth, C., Anael, Aubry, J. L., Bonnet, G., \& Duffield, Y. L. E.,M. (2014). Evidence of disturbed sleep and increased illness in overreached endurance athletes. Medicine \& Science in Sports Exercise; 46:1036-45. https://doi.org/10.1249/MSS.0000000000000177

Killer, S. C., Svendsen, I. S., Jeukendrup, A. E., \& Gleeson, M. Evidence of Disturbed Sleep and Mood State in Well-Trained Athletes During Short-Term Intensified Training with and Without a High Carbohydrate Nutritional Intervention. Journal of Sports Sciences; 35: 1402-1410. https://doi.org/10.1080/02640414.2015.1085589

Kölling, S., Wiewelhove, T., Raeder, C., Endler, S., Ferrauti, A., Meyer, T., \& Kellmann, M. (2015). Sleep monitoring of a six-day microcycle in strength and high-intensity training. European Journal of Sports Science; 10: 1-92015. https://doi.org/10.1080/17461391.2015.1041062

Lane, A. M., \& Terry, P. C. (2000). The nature of mood: Development of a conceptual model with a Focus on depression. Journal of Applied Sport Psychology; 12: 16-33. https://doi.org/10.1080/10413200008404211

Lastella, M., Lovell, G. P., \& Sargent, C. (2014). Athletes' precompetitive sleep behaviour and its relationship with subsequent precompetitive mood and performance. European Journal of Sports Science; 14, 123-130. https://doi.org/10.1080/17461391.2012.660505 
Research, Society and Development, v. 10, n. 14, e254101421896, 2021

(CC BY 4.0) | ISSN 2525-3409 | DOI: http://dx.doi.org/10.33448/rsd-v10i14.21896

McDowell, C. P., Campbell, M. J., \& Herring, M. P. (2016). Sex-related differences in mood responses to acute aerobic exercise. Medicine and Science in Sport and Exercise; 48: 1798-1802. https://doi.org/10.1249/MSS.0000000000000969

Melo, C. C., Pereira, J. K. C., Pachêco, T. N. P., Boletini, T. L., Belo, F. R. R., \& Noce, F. (2018). Os efeitos de uma prova de corrida nos estados de humor de praticantes amadores. Psicologia Revista; 27: 591-614. https://doi.org/10.23925/2594-3871.2018v27i3p591-614

Morgan, W. P. Test of champions: The iceberg profile. Psychol Today, 14: 92-108, 1980

Morgan, W. P. The trait psychology controversy. Res Q Exerc Sport 51: 50-76, 1980.

Nédélec, M., McCall, A., Carling, C., Legall, F., Berthoin, S., \& Dupont G. (2013). Recovery in soccer. Sports Medicine; 43(1):9-22. 10.1007/s40279-012$0002-0$

Ohayon, M., Wickwire, E. M., Hirshkowitz, M., et al. (2017). National Sleep Foundation's sleep quality recommendations: first report. Sleep Health; 3(1): 619. https://doi.org/10.1016/j.sleh.2016.11.006

Parsons-Smith, R. L., Terry, P. C., \& Machin, M. A. (2017). Identification and Description of Novel Mood Profile Clusters. Frontier in Psychology; 21:1958. https://doi.org/10.3389/fpsyg.2017.01958

Quartiroli, A., Parsons-Smith, R. L., Fogarty, G. J., Kuan, G., \& Terry, P. C. (2018). Cross-cultural validation of mood profile clusters in a sport and exercise context. Frontiers in Psychology; 9:1949. https://doi.org/10.3389/fpsyg.2018.01949

Rodrigues, D. F., Silva, A., Rosa, J. P. P., Ruiz, F. S., Veríssimo, A. W., Winckler, C., Rocha, E. A., Parsons, A., Tufik, S., \& Mello, M. T. (2017). Profiles of mood states, depression, sleep quality, sleepiness, and anxiety of the Paralympic athletics team: A longitudinal study. Apunts. Medicina de l'Esport; 52: 93-10. https://doi.org/10.1016/j.apunts.2016.11.002

Rohlfs, I. C. P. M. (2006). Validation of Brums test for mood evaluation in Brazilian athletes and non athletes. 111 f. Dissertação (Mestrado em Ciência do Movimento Humano) - Universidade do Estado de Santa Catarina, Florianópolis.

Teixeira, C. V. L. S., Ferreira, S. E., Evangelista, A. L., da Silva-Grigoletto, M. E., \& Gomes, R. J. Acute psychobiological responses of resistance training with different levels of social interaction. Rev Andal Med Dep 1: 1-5, 2016. 